\title{
Radiocarbon dating of samples collected during the 1984 expedition to North Greenland
}

\author{
Ole Bennike and Michael Kelly
}

The results of 18 radiocarbon age determinations on shells (14), plant material (2) and bone (2) are summarised here. The samples were collected during the GGU expedition to central and western North Greenland in 1984 (Henriksen, 1985). This list adds to the list published by Kelly \& Bennike (1985). In addition, a series of dates from Hall Land and Nyeboe Land has recently been published by England (1985).

The samples have been dated at the Carbon-14 Dating Laboratory of the Geological Survey of Denmark and the National Museum, Copenhagen (samples marked K), by courtesy of the Geological Survey of Denmark, at the Harwell Carbon 14/Tritium Laboratory, AERE, England (samples marked HAR) and at the Tandem Accelerator Laboratory in Uppsala (samples marked Ua). The samples from Uppsala have been dated by means of accelerator mass spectrometry (AMS) which has the advantage over conventional radiocarbon dating that very small samples can be dated (milligrams instead of grams).

The dates on marine shells from Copenhagen are corrected for isotopic fractionation by normalising to $\delta^{13} \mathrm{C}=0.0 \% \mathrm{PDB}$, whereas shell dates from Harwell and Uppsala are corrected by normalising to $\delta^{13} \mathrm{C}=-25 \% \mathrm{PDB}$. This difference in normalisation leads to a difference in age determination of $c .410$ years for samples with the same age (Mangerud, 1972). In order to obtain comparable ages, dates on marine shells have been corrected for ocean reservoir effect, following Funder (1982) for eastern North Greenland. Hence, shell dates normalised to $0.0 \%$ PDB have been corrected by -150 years, while shell dates normalised to $-25 \%$ PDB have been corrected by -550 years. This correction has been applied only to Holocene dates.

GGU 313804: K-4573. Wulff Land

$\mathbf{5 1 0 0 \pm 1 3 0 ~ B . P . ~} \delta^{13} C=-25.2 \%$ o

Basal sandy peat mainly composed of Carex stans and Salix arctica remains. The dating was carried out on Salix twigs to avoid contamination by intruding roots. Sample situated $275 \mathrm{~m}$ above sea level, $c$. $100 \mathrm{~m}$ from present margin of outlet glacier from the Inland Ice. South-western Wulff Land, $81^{\circ} 21^{\prime} \mathrm{N}, 48^{\circ} 55^{\prime} \mathrm{W}$.

GGU 313814: K-4373. Wulff Land

6780 \pm 105 B.P. $\delta^{13} C=+0.2 \%$ Corr. 6630

Shells of Arca glacialis, Hiatella arctica and Mya truncata from surface of laminated silt at $28 \mathrm{~m}$ above sea level. Gunnar Andersson Dal, northern Wulff Land, 82 $25^{\prime} \mathrm{N}, 50^{\circ} 31^{\prime} \mathrm{W}$.

GGU 313833: K-4374. Wulff Land

$8000 \pm 115$ B.P. $\delta^{13} C=+1.8 \%$ o Corr. 7850

Shells of Hiatella arctica and Mya truncata from surface of sand layer, which overlies folded silt and sand layers $62 \mathrm{~m}$ above sea level. Kap Wallén, eastern Wulff Land, $82^{\circ} 06^{\prime} \mathrm{N}$, $48^{\circ} 35^{\prime} \mathrm{W}$. 
GGU 313849: K-4375. Nyeboe Land

5530 \pm 95 B.P. $\delta^{13} C=+0.4 \% o$

Corr. 5380

Shells of Astarte borealis from surface of stony silt $28 \mathrm{~m}$ above sea level. Kap Brevoort, north-western Nyeboe Land, $81^{\circ} 56^{\prime} \mathrm{N}, 59^{\circ} 42^{\prime} \mathrm{W}$.

GGU 313858: HAR-6808. Hall Land

$>27500$ B.P. $\delta^{13} \mathrm{C}=+1.5 \%$

Shells of Hiatella arctica, Mya truncata and Astarte sp. from the surface of a moraine ridge at $227 \mathrm{~m}$ above sea level. Kap Sumner, northern Hall Land, $81^{\circ} 53^{\prime} \mathrm{N}, 60^{\circ} 35^{\prime} \mathrm{W}$.

GGU 313866: K-4376. Nyeboe Land

$4640 \pm 90$ B.P. $\delta^{13} C=-1.4 \%$ o Corr. 4490

Shells of Serripes groenlandicus from section in silt $13 \mathrm{~m}$ above sea level. Western Nyeboe Land, $81^{\circ} 42^{\prime} \mathrm{N}, 58^{\circ} 54^{\prime} \mathrm{W}$.

GGU 313877: K-4377. Hall Land

8080 \pm 115 B.P. $\delta^{I 3} C=+I .0 \%$ Corr. 7930

Shells of Mya truncata and Hiatella arctica from bottom of $3 \mathrm{~m}$ high silt section, underlain by diamicton $73 \mathrm{~m}$ above sea level. Atka Elv, western Hall Land, $81^{\circ} 35^{\prime} \mathrm{N}, 61^{\circ} 10^{\prime} \mathrm{W}$.

GGU 313892: HAR-6809. Hall Land

$>27500$ B.P. $\delta^{13} \mathrm{C}=+1.5 \%$

Plates of Balanus balanus and shells of Hiatella arctica from the surface of a moraine ridge at $139 \mathrm{~m}$ above sea level. Polaris Bugt, western Hall Land, $81^{\circ} 36^{\prime} \mathrm{N}, 61^{\circ} 20^{\prime} \mathrm{W}$.

GGU 313896: HAR-6745. Hall Land

$8870 \pm 100$ B.P. $\delta^{13} C=+1.3 \%$ o Corr. 8320

Shells of Hiatella arctica and Mya truncata from a thin layer of silt which overlies diamicton $89 \mathrm{~m}$ above sea level. 'Gråsten Elv', eastern Hall Land, $81^{\circ} 45^{\prime} \mathrm{N}, 59^{\circ} 35^{\prime} \mathrm{W}$.

GGU 313897: K-4687. Hall Land

$8680 \pm 120$ B.P. $\delta^{13} C=-16.6 \%$ Corr. 8530

Skeleton of Phoca hispida (ringed seal) from silt section $89 \mathrm{~m}$ above sea level. Collected close to GGU 313896. Date corrected for isotopic fractionation by normalising to $\delta^{13} \mathrm{C}=$ $0.0 \%$ PDB. 'Gråsten Elv', eastern Hall Land, $81^{\circ} 15^{\prime} \mathrm{N}, 59^{\circ} 36^{\prime} \mathrm{W}$.

GGU 313900: K-4378. Hall Land

8640 \pm 115 B.P. $\delta^{I 3} C=+1.9 \%$ o Corr. 8490

Shells of Hiatella arctica from surface of silt, $101 \mathrm{~m}$ above sea level. 'Gråsten Elv', eastern Hall Land, $81^{\circ} 48^{\prime} \mathrm{N}, 59^{\circ} 35^{\prime} \mathrm{W}$.

GGU 313914: K-4379. Hall Land

7740 \pm 115 B.P. $\delta^{13} C=+1.6 \%$ o Corr. 7590

Shells of Hiatella arctica and Mya truncata from section in silt and sand $76 \mathrm{~m}$ above sea level. Kap Ammen, northern Hall Land, $81^{\circ} 50^{\prime} \mathrm{N}, 61^{\circ} 24^{\prime} \mathrm{W}$. 
Shells of Hiatella arctica from surface of fine sand and silt $71 \mathrm{~m}$ above sea level. Th. Thomsen Fjord, Nansen Land, $83^{\circ} 03^{\prime} \mathrm{N}, 43^{\circ} 24^{\prime} \mathrm{W}$.

GGU 313932: HAR-6746. Nansen Land

$6100 \pm 100$ B.P. $\delta^{13} C=+1.7 \%$ Corr. 5550

Shells of Hiatella arctica from surface of frost disturbed silt at $11 \mathrm{~m}$ above sea level. Kap Ramsay, northern Nansen Land, $83^{\circ} 10^{\prime} \mathrm{N}, 44^{\circ} 42^{\prime} \mathrm{W}$.

GGU 313935: Ua-323. Nyeboe Land

$2155 \pm 90$ B.P. $\delta^{13} C=-25.0 \%$

Metacarpi of Rangifer tarandus (reindeer) from surface of ground. Identified and submitted by M. Meldgaard, Zoological Museum, Copenhagen. Mouth of Alma Glück Dal, southeastern Nyeboe Land, $81^{\circ} 27^{\prime} \mathrm{N}, 54^{\circ} 50^{\prime} \mathrm{W}$.

GGU 313940: K-4380. Nyeboe Land

4870 \pm 80 B.P. $\delta^{13} C=+2.3 \%$ o

Corr. 4720

Shells of Hiatella arctica and Mya truncata from surface of silt, $24 \mathrm{~m}$ above sea level. Bottom of Sankt George Fjord, south-eastern Nyeboe Land, $81^{\circ} 28^{\prime} \mathrm{N}, 54^{\circ} 39^{\prime} \mathrm{W}$.

GGU 313944: K-4574. Warming Land

$32270+{ }_{-1200}^{+1120}$ B.P. $\delta^{13} C=+27.5 \%$ o

Allochthonous plant detritus from section in lacustrine, laminated silt and fine sand, 115 $\mathrm{m}$ above sea level. Skalhøjene, northern Warming Land, $81^{\circ} 57^{\prime} \mathrm{N}, 53^{\circ} 38^{\prime} \mathrm{W}$.

GGU 313949: K-4381. Wulff Land

5180 \pm 90 B.P. $\delta^{13} C=+0.1 \%$ Corr. 5030

Shells of Hiatella arctica and Mya truncata from surface of low sand ridge $c .5 \mathrm{~m}$ above sea level. Røhling Land, south-eastern Wulff Land, $81^{\circ} 50^{\prime} \mathrm{N}, 46^{\circ} 56^{\prime} \mathrm{W}$.

\section{References}

England, J. 1985: The late Quaternary history of Hall Land, northwest Greenland. Can. J. Earth Sci. 22, 1394-1408.

Funder, S. 1982: C-dating of samples collected during the 1979 expedition to North Greenland. Rapp. Grønlands geol. Unders. 110, 9-14.

Kelly, M. \& Bennike, O. 1985: Quaternary geology of parts of central and western North Greenland: a preliminary account. Rapp. Grønlands geol. Unders. 126, 111-126.

Mangerud, J. 1972: Radiocarbon dating of marine shells, including a discussion of apparent age of recent shells from Norway. Boreas 1, 143-172.

O. B.,

Geologisk Museum.

DK-1350 København $K$,

Denmark.
M. K., Department of Environmental Science, University of Lancaster, Lancaster LAI $4 Y Q$, 